\section{El talento humano como factor de competitividad en la actividad turística en Lima Metropolitana}

\author{
Human talent as competitive factor at touristic \\ activity in Metropolitan Lima
}

\begin{abstract}
RESUMEN
El turismo ha venido creciendo en nuestro país y, específicamente en la ciudad de Lima Metropolitana, puesto que alberga a una parte significativa de las diversas empresas de servicios turísticos: agencias de viajes y tour operadoras, establecimientos de hospedajes, empresas de transporte turístico, restaurantes turísticos, etc. Las empresas turísticas como toda organización están constituida por personas, convirtiéndose en el recurso más valioso de la organización. Nuestro objetivo fue el análisis del talento humano en base al desarrollo de sus competencias, habilidades, capacitación continua, incentivos y reconocimientos, puesto que son los protagonistas y líderes en el alcanzar las metas u objetivos de la empresa. Como principal conclusión encontramos que a pesar que el talento humano es un factor de competitividad aún no se reconoce la importancia, ya que todavía no se potencializa al talento humano debidamente en los objetivos organizacionales de la empresa.
\end{abstract}

Palabras claves: Talento humano; competitividad; actividad turística.

\section{ABSTRACT}

Tourism has been growing in our country, specifically at Metropolitan Lima since it houses a significant part of touristic services: travel agencies and tour operators, lodgings, touristic transportation, touristic restaurants, etc. Touristic companies as every single organization is made up of people, becoming the most value resource in the organization. Our objective was to analyze human talent based on its competences, abilities, updated training, incentives y recognitions, because they are the starring and leaders in achieving the business's objectives and goals. As main conclusion, it was founded that even though human talent is a competitive factor, it is not recognizable its importance since it is not potentiated in the organizational objectives of the company.

Keywords: Human talent; competitiveness; touristic activity .

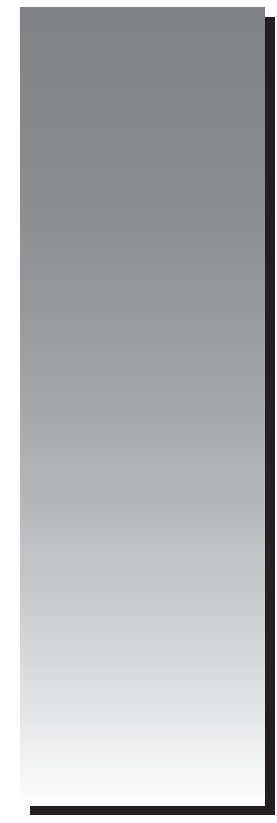

Teresa Jesús Ríos Delgado

triosd@unmsm.edu.pe

Edelmira del Rosario Mendoza Púa ${ }^{1}$

emendozap@unmsm.edu.pe

${ }^{1}$ Universidad Nacional Mayor de San Marcos, Facultad de Ciencias Administrativas. Lima, Perú 


\section{INTRODUCCIÓN}

El turismo es una actividad que involucra personas, que buscan experiencias únicas y que se relacionan con personas que ofrecen el servicio en diversos destinos turísticos. Así mismo, dicha actividad involucra a diversas empresas que ofrecen servicios, necesarios e importantes para el cumplimiento de actividad en sí. De tal manera que la participación del factor humano resulta ser imprescindible. El objetivo general fue analizar el talento humano como factor de competitividad en la actividad turística en Lima Metropolitana y como objetivos específicos el establecer la segmentación del personal profesional y técnico en turismo en Lima Metropolitana e identificar los productos y servicios de la oferta turística en Lima Metropolitana. Si bien es cierto durante el proceso de negociación, venta y toma del servicio se puede hacer uso de la tecnología es, claro que el talento humano siempre será necesario para cerrar cualquier tipo de operación. Podríamos cuestionar ¿por qué? y, la respuesta es que a diferencia de vender cualquier otro producto, en la actividad turística se venden sueños, sensaciones, emociones, experiencias. Por lo tanto, el factor imperante que toda organización debe lograr las competencias en la gestión de los recursos humanos. A pesar de todas las investigaciones previas y teorías consolidadas sobre este tema, aún hay sectores empresariales que no han prestado atención a una gestión eficaz y lograr competitividad o por lo menos construir las bases. Las empresas turísticas necesitan de personas que respondan no solamente a los objetivos sino también a un beneficio mutuo. Además, los continuos cambios o transformaciones en esta área -especialmente en la tecnología, también exigen continuar siempre en la búsqueda de los nuevos aportes del talento humano a las organizaciones.

En la tesis sobre competitividad en el sector turismo en Colombia (Bravo \& Rincón, 2013) se establecen las siguientes conclusiones:

Finalmente, es importante concluir, que la competitividad en el sector turismo colombiano ha logrado vencer paradigmas mejorando la eficiencia en el uso de los recursos económicos, físicos y humanos, sin embargo el país no puede dejar de invertir esfuerzos en estos proyectos ni puede descuidar lo que se ha conseguido hasta el momento ya que será precisamente cuando se establezca estas prácticas como una cultura de pensamiento y forma de vida, que Colombia logrará posicionarse como un destino turístico de talla mundial.

Según los autores Colombia ha mejorado en el uso eficiente de entre todos los que mencionan los recursos humanos y, ese cierto ya que en esta parte de la región sur. Colombia es un referente respecto al desarrollo de la actividad turística, país que pese a sus conflictos internos que han sufrido durante muchos años ellos han venido desarrollando la actividad turística enfocadas al desarrollo del profesional en cualesquiera de las ramas de la actividad, tal es así que están desarrollando programas para un sector de la oferta como son los discapacitados, teniendo como visión el desarrollo del turismo social y para ello se ocupan de los colaboradores que trabajan en los hoteles y en otras áreas del sector.

Otro antecedente es Bolaky (2011) en su artículo "La competitividad del turismo en el Caribe" se plantean las siguientes conclusiones:

Para mantener o mejorar su competitividad turística, los destinos caribeños deberán ser también más competitivos en costos y precios. Eso puede requerir un apoyo al desarrollo del sector privado local con el propósito de reducir las fugas de importaciones y crear vínculos entre el sector del turismo y el resto de la economía; la disminución del consumo del gobierno para mantener tipos impositivos competitivos; la reducción de la vulnerabilidad a los desastres naturales; la reforma de los mercados laborales y de las normas comerciales en general; el mantenimiento de un entorno saludable y seguro; la inversión en desarrollo humano y en tecnología; el desarrollo de una política de transportes o aviación que permita aminorar los costos de transporte a la región y desde esta, y una ampliación del acceso por vía aérea.

El autor manifiesta que existe por los menos dos variables bajo las cuales se puede establecer la competitividad turística en los países del Caribe. Si bien es cierto el Caribe posee una oferta turística de sol y playa, también ofrece 
naturaleza, ecoturismo y aventura a pesar de estar expuesta a los desastres naturales, pero el factor fundamental es el recurso humano por el cual tanto las políticas salariales y laborales deberán mejorar.

En el artículo "La competitividad turística: imperativo para Manzanillo, Colima" (Amaya, Conde, \& Covarrubias, 2008), se plantean las siguientes conclusiones:

Para competir en el ámbito turístico, un destino, además de ventajas comparativas, precisa de ventajas competitivas, es decir, no sólo es necesario poseer una variedad más o menos amplia de productos y recursos turísticos, sino que éstos deben ser gestionados de forma eficiente y eficaz a mediano y a largo plazo.

Asimismo, del análisis de los principales modelos de competitividad turística encontrados en la literatura hay muchos factores que influyen en la competitividad de un destino turístico.

\section{El talento humano y la competitividad.}

Toda organización está constituida por personas, o personal cuya complejidad de su comportamiento, desempeño, metas u objetivos, etc. siguen influyendo o condicionando a la organización en su desarrollo. El escenario entonces se enfoca en potenciar los recursos humanos en favor de la organización, estableciendo sus objetivos organizacionales, sea de producción o prestación de servicios.

Efectivamente la persona se convierte en un en generadora de valor de la organización, incrementándose la productividad y/o mejorando el servicio al cliente, lo que conlleva finalmente a obtener competitividad.

El talento humano es un término que se utiliza en estos tiempos donde la tecnología es imperante en diario quehacer de la personas y exige una diversificación o ampliación de ciertas capacidades, aptitudes, actitudes para realizar actividades en general. Y la organización y empresa no está exenta de esta. Hoy donde la máquina sigue reemplazando al hombre y los conflictos por la cantidad de datos, información o conocimiento debe estar a la par con la experiencia, en el logro del éxito de la empresa, en una eficiencia y eficacia de la organización.
Ya es reconocido que el talento humano es un elemento de la competitividad, - ya no es solamente el trabajo o actividad que la persona uno ejerce, sino el papel intangible dentro de la organización. Y en estas décadas convulsionadas por los avances tecnológicos y dependencia (a veces peligrosa) de las redes sociales, se debe estar en constante evaluación o revaluación de los objetivos de la organización, pero sobre todo del papel del colaborador como fuente de competitividad.

La competitividad se define por la productividad con la que un país utiliza sus recursos humanos, económicos y naturales. Para comprender la competitividad, el punto de partida son las fuentes subyacentes de prosperidad que posee un país. El nivel de vida de un país se determina por la productividad de su economía, que se mide por el valor de los bienes y servicios producidos por unidad de sus recursos humanos, económicos y naturales. La productividad también depende de la capacidad de una economía para movilizar sus recursos humanos disponibles. (Porter, 2002).

Entonces, uno de los baluartes de la competitividad es el recurso humano; tal como (Gubman, 2000) lo menciona al considerar que es el único elemento necesario y suficiente para ejecutar la estrategia, de tal manera que los trabajadores calificados le pertenecen una gran parte de su éxito futuro, incluida su fortuna personal, valorándose a los colaboradores. Efectivamente, la valoración de la persona, del colaborador, del empleado es la piedra angular de la valoración de la organización. Tanto la organización como sus colaboradores forman una relación biunívoca de desarrollo y bienestar. "El talento humano se ha convertido en una fuente crucial de ventaja competitiva. Las organizaciones que lo hagan mejor en cuanto a atraer, desarrollar, entusiasmar y retener a los más aptos, ganarán más y mejorarán grandemente su desempeño" (Michaels, Handfield, \& Axelrod, 2003). Situándonos en la actividad turística, hemos identificados cinco características del talento humano como elemento de competitividad:

\section{Compensaciones.}

Generalmente cuando se utiliza el término compensaciones, nos referimos a los sueldos, salarios, 
incentivos, bonificaciones y prestaciones, etc. (retribución) que se pagan a los colaboradores por un servicio prestado o actividad desarrollada.

Toda organización requiere el talento humano para lograr sus objetivos organizaciones y este talento humano busca ser recompensado en la colaboración, aporte, identificación que realiza con la organización. Entonces, estamos hablando de sueldos, salarios, y hasta gratificaciones que los colaboradores reciben por su trabajo.

El salario de los empleados en relación con otras oportunidades laborales alternativas influye directamente en la capacidad de la empresa para atraer trabajadores de otras empresas. La satisfacción con el salario tiene una alta correlación con el nivel salarial, siendo la insatisfacción con el salario uno de los mejores predictores de la rotación de trabajadores. (Gómez-Mejía, Balkin, \& Cardy, 2008).

Por lo cual, la organización debe garantizar:

La satisfacción de los empleados, lo que a su vez ayuda a la organización a obtener, mantener y retener una fuerza de trabajo productiva. El deseo de obtener mejor compensación puede disminuir el desempeño, incrementar el nivel de quejas o conducir a los empleados a buscar un empleo diferente. (Jiménez \& Hernández, 2010).

Y como lo plantea Chiavenato para mejorar el desempeño, las personas deben percibir justicia en las recompensas que reciben. Recompensar los buenos resultados y no recompensar a las personas que no tienen un buen desempeño, los objetivos deben ser claros, así como el método para medirlos (Chiavenato, 2009).

\section{Motivación.}

En general, es capacidad de realizar las actividades más allá por encima de lo convencional o de lo establecido para mejorar e incrementar los resultados, productividad, réditos, etc. Muy ligado entonces está la motivación, que podría decirse que es la recompensa el desempeño para motivar el mismo. Impulsando un buen desempeño se puede establecer una armonía entre la satisfacción y la compensación dada y la capacidad de competitividad de la organización. La motivación es el deseo del colaborador de "hacer el trabajo lo mejor posible, o de ejercer más esfuerzo para realizar las tareas encomendadas" (Gómez-Mejía, Balkin, \& Cardy, 2008). Hay motivaciones intrínsecas que surgen de la identificación y/o valoración del colaborador hacia la organización o también extrínsecas cuando la organización se identifica y/o valora al colaborador, por ejemplo, a través de las compensaciones, premios, etc. Chiavenato también enfatiza en "dar reconocimiento a las personas y no solo dinero constituye el elemento básico de la motivación humana" (Chiavenato, 2009).

\section{Desarrollo y Promoción.}

El desarrollo del recurso humano se debe sustentar en diversas actividades que permitan formular planes de formación, capacitación, mejora continua, etc. y de esta manera potenciar a los colaboradores, motivarlos, controlar el desempeño, fomentar la participación y por su puesto evitar o disminuir el absentismo o ausentismo en la práctica. La organización, entonces, debe elaborar procesos continuos y simultáneos de capacitación dirigidos a alcanzar diversas habilidades, destrezas, y valores por parte de los colaboradores. Lo cual se va a ver reflejado en el desempeño, garantizando la formación del colaborador durante su vida laboral. Por otro lado, la organización debe brindar al colaborador una retroalimentación en relación a su desempeño como fundamento de aumentos de salario, bonificaciones, promociones, incentivos hasta el despido, en algunos casos.

\section{Comunicación.}

Según Barreiro (2000):

La comunicación es un proceso de amplia relevancia en el funcionamiento de cualquier grupo, organización o sociedad, de ahí que, en la teoría de la organización, la comunicación juega un lugar central, ya que la estructura, tamaño y alcance de la organización están casi totalmente determinadas por la comunicación.

Igualmente, Porter (2002) afirma que "lo que hace a una empresa competitiva, es su capacidad para crear productos o servicios con valor para el cliente y esto solo lo logran las empresas configurando su cadena de valor".

La comunicación consiste en la transferencia y comprensión de información de una persona a otra. Constituye un puente de 
transferencia entre las personas, para que puedan compartir lo que sienten y saben. Cuando se maneja el proceso en forma adecuada se obtiene una comunicación abierta. La comunicación abierta se presenta cuando las personas se sienten libres de comunicar todos los mensajes relevantes. Sin ella la cooperación de torna prácticamente imposible, porque las personas no pueden comunicar sus pensamientos, necesidades y sentimientos a las otras. Puede afirmarse que todo acto de comunicación influye de alguna manera a la organización (Koontz \& O’Donnell, 2000).

\section{Adaptación al Puesto de Trabajo.}

Esta última característica que se está considerando implica que la organización debe establecer de integración de personal con el sistema administrativo. Por ejemplo, los propósitos de una empresa son los propósitos de la organización, necesarios en el logro de los objetivos. La estructura organizacional establece, por ejemplo, la cantidad, las características, capacidades, competencias, etc., de gerentes que se necesitan, definiéndose el perfil puesto, cargo o perfil. Y en base a los objetivos organizaciones se formulan las capacitaciones, actualizaciones, los procesos de reclutamiento, selección, ocupación, promoción y hasta despidos o jubilaciones. Recordemos el planteamiento de (Drucker, 2002) que el desarrollo del talento de la gente es la tarea más importante del negocio. Por lo cual, cuando se accede a un puesto de trabajo, el cambio o variación del elemento interpersonal de los futuros colaboradores, exige una evaluación preliminar del puesto de trabajo y de los compromisos o características del mismo, siempre con la consideración no solamente que encaje en el puesto de trabajo si no también que evolucione como colaborar, socio estratégico, y en beneficio de la organización.

\section{La actividad turística en Lima Metropo- litana.}

Schwab (2015) dice:

El Perú posee un gran potencial de desarrollo turístico. Al respecto, el WEF advierte la gran variedad de paisajes, fauna, la cultura y la historia como principales atractivos que el Perú ostenta, pero al mismo tiempo que señala como desafío la expansión de la actividad turística bajo estándares de sostenibilidad).

Según la Organización Mundial del Turismo (2016):

Pese a los altibajos de la economía global, el turismo ha experimentado un crecimiento prácticamente ininterrumpido. Las llegadas de los turistas internacionales a escala mundial han pasado de 25 millones en 1950 a 278 millones en 1980 , 527 millones en 1995 y 1.133 millones en el 2014. De forma análoga, los ingresos mundiales por turismo internacional obtenidos han pasado de US $\$ 2.000$ millones de dólares en 1950 a US $\$ 104.000$ millones en 1995 y US\$1.425.000 millones en el 2014.

Lima está ubicada en la vertiente occidental de los andes peruanos, en el centro del territorio nacional. Su importancia radica a que en su suelo vive cerca de un tercio de la población peruana, se genera más del $50 \%$ de la producción de bienes y servicios del país y es el centro político por excelencia (Instituto Nacional de Estadística e Informática, 2001) - lamentablemente no se ha encontrado datos más actualizados. En la planta turística de Lima Metropolitana, se puede encontrar el hospedaje que pueden ser clasificados en hoteles, apart-hoteles, hostales y albergues. También están los restaurantes, que son categorizadas especialmente por la calidad de las instalaciones, equipos y servicios. Por ejemplo, existen restaurantes de 1 tenedor, 2 y 3 tenedores y los de primera categoría que son de 4 y 5 tenedores. Igualmente, las agencias de viaje también participan directamente en la comercialización del Perú como destino turístico. Cabe destacar que según (INEI, 2014) existen aproximadamente cerca de 1,500 restaurantes, 130 agencias de viajes, 75 establecimientos de hospedaje y 170 establecimientos de artesanía, lo cual indica que la actividad turística en Lima es dinámica y está dirigida principalmente al turismo receptivo. Debemos agregar la gran influencia de los factores políticos, económicos, demográficos y en especial del tecnológico, los cuales exigen nuevas necesidades, nuevos productos, pero sobre todo un nuevo turista con llega a nuestro país con un diferente estilo de vida e interés. 


\section{MÉTODOS}

El presente estudio es de tipo descriptivo porque busca detallar las características y/o indicadores y los perfiles de la(s) persona(s), o comunidades con los cuales se desarrolló esta investigación. Las técnicas aplicadas en este estudio fueron las siguientes: el análisis documentario que se llevó a cabo a través de guías de observación (análisis de contenido) mediante el cual se recopiló la información; una encuesta; y el fichaje. La población estuvo constituida por las empresas de agencias de viaje (AAVV), hospedajes y restaurantes de Lima Metropolitana, sin embargo, por disponibilidad en la participación solo se logró encuestar a 56 colaboradores de estas diversas empresas. Una vez recopilada la información, se elaboraron cuadros estadísticos y gráficos a fin de facilitar el análisis descriptivo de cada una de ellas utilizando el método inductivo-deductivo.

\section{RESULTADOS}

Los resultados obtenidos del trabajo de campo fueron analizados a nivel descriptivo e inferencial:

En relación al tema de compensaciones de la organización, se puede apreciar cierto consenso entre los participantes sobre el papel que juega la organización al identificarse con su colaborador, lo cual ratifica que efectivamente la percepción del colaborador con respecto a las políticas de compensación de la organización está altamente relacionada a la productividad. Además de ello se percibe que a pesar de que existe una preocupación por la empresa aún podría decirse que no está definido la consideración de "socio" al colaborador.

Respecto a la motivación por parte de la organización, se puede apreciar una mayor motivación en el profesional y el estudiante; podría decirse que la labor técnica aún no está definida como "motivante". Sin embargo, sigue siendo importante la motivación sea cualitativa o cuantitativa. Respecto a la motivación de valoración cualitativa resulta muy significativa debido a que las personas siempre esperan la demostración de aprecio a su labor, este aprecio está dirigido fundamentalmente a su autoestima.
Acerca del desarrollo y promoción, se puede apreciar un consenso en la identificación de la organización con sus colaboradores. Sin embargo, el factor personal, llámese relaciones interpersonales, sigue siendo un tema que aún no forma parte de la política de la organización, lo cual también se puede observar en algunos colaboradores al responder que no hay una alta relación con los objetivos de la empresa.

La percepción del colaborador respecto al desarrollo y promoción en la empresa esta significativamente en relación a las políticas de la empresa, sin embargo, no así en relación al factor personal del colaborador. Aunque parezca contradictorio el colaborador sabe que la organización se esfuerza en el desarrollo de ellos por ser un elemento importante para la productividad, pero no en si por ellos mismo como factor humano, ello podría justificar la falta de identidad que la empresa no logra obtener de los colaboradores.

En relación a la comunicación, si bien hay un consenso sobre el valor de la comunicación en una organización, se puede apreciar que es el profesional quien más enfatiza este tema. Ello puede estar en relación a que el profesional se forma para dirigir grupos y una herramienta fundamental de gestión es el desarrollo de la comunicación eficaz, está obligado a iniciar la comunicación y a desarrollarla de forma asertiva.

Respecto a la adaptación al puesto de trabajo, se puede apreciar un consenso en la disponibilidad de los colaboradores en seguir aprendiendo, mejorando en sus labores lo cual les permite visualizar sus aspiraciones a largo plazo. Con lo cual se evidencia que la empresa muestra preocupación por hacer que los colaboradores puedan desarrollarse en las áreas de su competencia.

En la figura 1 se muestra la especialidad de los entrevistados en las empresas de servicios turísticos en Lima Metropolitana.

Finalmente, respecto a la segmentación del personal según su especialidad, el gráfico muestra que el 50\% resulta siendo profesional y un $30,4 \%$ es técnico en turismo, así mismo existe un $17,9 \%$ que es estudiante en algunos casos pueden deberse a que son practicantes. 


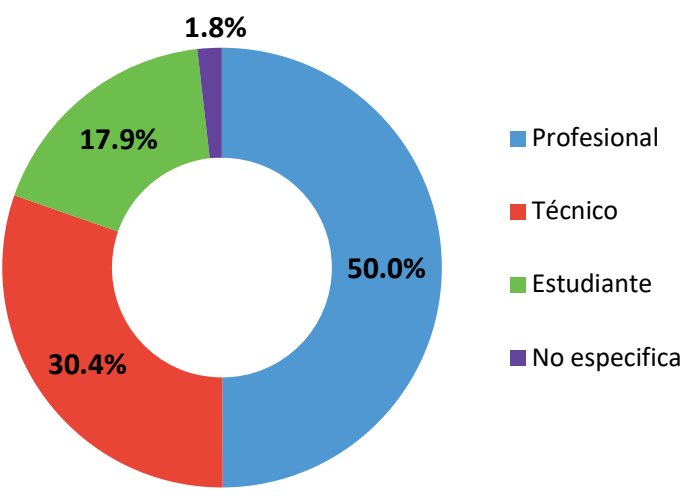

Figura 1. Porcentaje de entrevistados según su especialidad.

Fuente: Elaboración propia

\section{DISCUSIÓN}

El objetivo general de este estudio fue el analizar el talento humano como factor de competitividad en la actividad turística en el área de Lima Metropolitana. A pesar de los pocos estudios en el área de turismo y en base a la bibliografía consulta, podemos observar que el recurso humano como factor de competitividad es muy importante, resaltando que se mejora la eficiencia en el uso de los recursos económicos, físicos y humanos (Bravo \& Rincón, 2013).

En base a nuestros hallazgos coincidimos con (Gubman, 2000) cuando se refiere al recurso humano como el único elemento necesario y suficiente para ejecutar la estrategia, de tal manera que los trabajadores calificados le pertenecen una gran parte de su éxito futuro, incluida su fortuna personal.

Otro de los objetivos fue establecer la segmentación del personal profesional y técnico en turismo en Lima Metropolitana, la cual a pesar de la poca participación de la población, el $50 \%$ son profesionales, un dato muy aliciente en el área de turismo, puesto que además del boom gastronómico, tenemos mucho más que ofrecer como destino turístico, por lo cual es preponderante que el profesional del turismo sea el protagonista en esta empresa, en segundo lugar está personal técnico, en general por cuestiones de remuneraciones -principalmente. Es así que se deja de lado, por ejemplo, lo que plantea (Michaels, Handfield, \& Axelrod, 2001) que el talento humano es una factor transcendental de la ventaja competitiva.
A pesar que el tema de talento humano en turismo es bastante álgido, de tal manera que la participación del factor humano resulta ser imprescindible, no hay un interés genuino en invertir (sea económico y/o social) en ello. Si bien es cierto durante el proceso de negociación, venta y toma del servicio se puede hacer uso de la tecnología es, claro que el recurso humano siempre será necesario para cerrar cualquier tipo de operación. Podríamos cuestionar ¿por qué? y, la respuesta es que, a diferencia de vender cualquier otro producto, en la actividad turística se venden sueños, sensaciones, emociones, experiencias. Sin duda el presente trabajo muestra la percepción del personal respecto de la empresa de servicios turísticos en Lima Metropolitana. Además de ello muestra una realidad incongruente con una Ciudad catalogada como Patrimonio de la Humanidad de la que poca información se tiene a pesar de ser el centro de la actividad turística en nuestro país. Es necesario conocer más de ella respecto del desarrollo de las empresas turísticas en cuanto al manejo del recurso humano y de la propia organización. La información que se obtiene de Lima Metropolitana pasa por la información que se recoge como Lima Provincia. La relación de las empresas que figura en la información del MINCETUR no se encuentra actualizada, y se comprobó una vez que se tuvo que aplicar la encuesta que, muchas de las direcciones no correspondían. Así mismo respecto a la información de empresas que registra INEI no son datos actualizados. Por lo que en este aspecto aún se tiene que trabajar mucho a fin de conocer que tipos de empresas de servicios turísticos operan en el Centro Histórico de Lima, a fin de constatar cual es la cadena de valor que desarrolla la actividad turística, dado que la oferta de recursos y/o atractivos está orientado al desarrollo de un turismo receptivo esencialmente.

\section{CONCLUSIONES}

El turismo es una actividad de servicios, es por ello que para la actividad turística el talento humano es un elemento primordial y básico en el desarrollo de la propia actividad. Por lo cual, es importante en el sector turismo establecer políticas de compensaciones de acuerdo a su productividad (ventas de paquetes turísticos, ventas de 
pasajes aéreos, terrestres, venta de habitaciones, venta de servicios en restaurantes, etc.).

En cuanto al reconocimiento del talento humano en el sector turismo, como en cualquier empresa, esta no puede estar ausente $y$, por tanto, tiene una valoración tanto cualitativa como cuantitativa. Este sector trata y se involucra directamente con personas $\mathrm{y}$, no se puede dejar de reconocer su labor.

El desarrollo y promoción debe estar vinculado directamente al aspecto personal. Al ser grupos humanos diversos, una promoción, un reconocimiento a su labor resulta mucho más significativo que cualquier otra forma otorgada.

La organización sustenta su labor en la comunicación dado que es un aspecto esencial, básico y necesario en todo tipo de empresa. Y, el sector turismo no es ajeno a ello, ya que tratamos con personas de diversos grupos heterogéneos y culturas diversas.

Así mismo siendo una actividad que está muy relacionada a grupos diversos y al uso de tecnología, está sometida a constantes cambios. Esto conlleva a que el personal que trabaja en este sector esté dispuesto y acostumbrado a las diversas acciones de desarrollo que la empresa pueda otorgarle.

A pesar que el talento humano es un factor de competitividad en la empresa turística, aun no se reconoce la importancia como capacidad competitiva, ya que aún no se potencializa al factor humano debidamente en los objetivos organizacionales de la empresa.

Si bien se reconoce el talento de los colaboradores, son muy pocas las empresas que los consideran o los convierten en socios estratégicos para lograr sus objetivos. No se observa una política de desarrollo al talento humano, ello se refleja a través de los resultados que muestran que la percepción del colaborador es aceptable en relación a los logros de la empresa no así en relación a aspectos personales.

En este vertiginoso avance de la ciencia, en donde las redes sociales son un canal y una fuente de información, lo que se busca es valorar, reconocer y motivar al colaborador desde lo más básico como el salario, lugar de trabajo, pasando por la oportunidad de hacer carrera en la organización y finalmente llegar a trascender en la sociedad, a través del reconocimiento, por ejemplo, por parte de la organización o sociedad.

\section{REFERENCIAS BIBLIOGRÁFICAS}

Amaya Molinar, Carlos Mario; Conde Pérez, Ernesto Manuel; Covarrubias Ramírez, Rafael (2008). La competitividad turística: Imperativo para Manzanillo, Colima. Teoría y Praxis. pag. 17 32. Universidad de Colima, Escuela de Turismo. México.

Barreiro, L. (2000). La Comunicación en la administración empresarial. $\mathrm{N}^{\circ} 2$. Revista Espacio. Cuba.

Bolaky, Binesware (2011). La competitividad en el Caribe. Revista CEPAL 104. Agosto pag. 59 - 79.

Bravo Montilla, Ángela María \& Rincón Ambrosio David Ernesto. (2013). Estudio de competitividad en el sector turismo en Colombia, tesis del programa de administración de negocios internacionales. Universidad del Rosario, Facultad de Administración Bogotá d.c.

Chiavenato, I. (2009) Gestión del Talento Humano. México. Mc Graw Hill

Drucker, P. (2002). La Gerencia en la Sociedad Futura. Bogotá. Editorial NORMA

Gómez-Mejía,L; Balkin,D \& Cardy, R. (2008). Gestión de Recursos Humanos. Pearson

Gubman, E. (2000). El Talento como Solución. Bogotá: McGraw-Hill

INEI (2001). Conociendo Lima. Guía Estadística. Lima. Dirección Nacional de Estadística e Informática Departamental

INEI (2014). Una mirada a Lima Metropolitana. Lima. Dirección Nacional de Estadística e Informática Departamental

Jiménez Ramírez, L \& Hernández Ovalle, S (2010). Importancia de la Compensaciones laborales y de la gestión humana en las empresas. Recuperado el 04 de Diciembre de 2018, de https://revistas. uexternado.edu.co/index.php/proyecta/article/download/1958/1748/

Koontz \& O’Donnell (2000). Administración. 8a ed. México. Mc Graw Hill.

Michaels, E G, Handfield-Jones, H \& Axelrod, B (2001) The War for Talent. Harvard Business School Press, Boston, MA

OMT (2016). Panorama OMT del turismo internacional. Recuperado el 23 de noviembre de 2018 de https://www.e-unwto.org/doi/ pdf/10.18111/9789284418152 
El talento humano como factor de competitividad en la actividad turística en Lima Metropolitana

Porter, M. (2002). Ventaja Competitiva. México, ALAY EDICIONES

Schwab, K (2015). The Global Competitiveness Report 2013-2014. World Economic Forum 